Cuadernos de Filología Italiana

ISSN: 1133-9527

http://dx.doi.org/10.5209/CFIT.57968

\title{
Il teatro ilarotragico di Manganelli (tra Beckett e Bacon)
}

\author{
Filippo Milani ${ }^{1}$
}

Ricevuto 13 novembre 2018: / Modificato: 03 marzo 2018 / Accettato: 22 maggio 2018

Riassunto. La ricezione italiana dell'opera di Samuel Beckett, seppur tardiva, trova in Giorgio Manganelli uno degli interpreti più attenti e puntuali. Un aspetto che merita di essere approfondito riguarda il carattere ilarotragico del teatro beckettiano secondo l'interpretazione di Manganelli, ovvero l'operazione di svuotamento del mito tragico in funzione parodica. Le "tragedie da leggere" di Manganelli condividono con le opere teatrali di Beckett e con le tele di Bacon il medesimo intento dissacratorio nei confronti del mito tragico, a cui si oppone una "ilarità" sarcastica e malinconica.

Parole chiave: Manganelli; Beckett; Bacon; teatro dell'assurdo; vocalità in scena.

\section{[en] Manganelli's hilarious-tragic theatre (between Beckett and Bacon)}

\begin{abstract}
The Italian reception of Samuel Beckett's works, even though late, finds in Giorgio Manganelli one of the most careful and punctual interpreters. An aspect that is necessary to analyze concerns the hilarious-tragic character according to Manganelli's interpretation, or rather the operation of emptying the tragic myth in a parodic way. The Manganelli's "tragedie da leggere" have in common with Beckett's plays and with Bacon's paintings the same desecrating purpose towards the tragic myth, which they oppose a sarcastic and melancholic hilarity.
\end{abstract}

Key words: Manganelli; Beckett; Bacon; theatre of absurd; voices on stage.

Come citare: Milani, Filippo (2018) «Il teatro ilarotagico di Manganelli (tra Beckett e Bacon)», Cuadernos de Filología Italiana, 25, pp. 197-211.

La ricezione italiana dell'opera di Samuel Beckett, seppur tardiva e soggetta a frettolose classificazioni, trova in Giorgio Manganelli uno degli interpreti più attenti e puntuali ${ }^{2}$. Un aspetto che merita di essere approfondito riguarda l'operazione di svuotamento del mito tragico in funzione parodica caratteristico del teatro beckettiano, che Manganelli definisce come carattere ilarotragico, in un malinconico ribaltamento tra comicità e tragedia. In merito a tale "degradazione" del tragico Manganelli ha scritto, in un articolo su Murphy del 1962 - anno della pubblicazione della prima traduzione italiana da parte di Franco Quadri per Einaudi -, che «Beckett è un grande rètore, esperto a destare onesti e immaginosi terrori, fantastico inventore di inferni senza Dio al centro dei quali ha collocato la solitaria, orrenda e intelligente

\footnotetext{
1 Università di Bologna. Dipartimento di Filologia Classica e Italianistica, via Zamboni 32, 40126 - Bologna. filippo.milani@unibo.it

2 V. la raccolta di saggi Alfano / Cortellessa (2006); inoltre Alfano (2010), Baldacci (2010) e Scarlini (1996).
} 
risata dell'uomo “pericoloso a sé e agli altri”» (Manganelli 1962 [2004]: 102). L'opera dello scrittore irlandese si caratterizza per la spiccata capacità di saper costruire luoghi desolatamente vuoti attraverso minuziose strategie retoriche, grazie alle quali si rivela tutta la carica evocativa e perturbante del linguaggio, svelando all'uomo la propria innata volontà di auto-distruzione, sia fisica che verbale. Le "tragedie da leggere" di Manganelli - emblematico titolo della raccolta di testi teatrali a cura di Luca Scarlini - sono pervase dal medesimo intento dissacratorio nei confronti del mito tragico, a cui si oppone una ilarità sarcastica e malinconica.

In questa prospettiva, ritengo che sia possibile estendere a ritroso la definizione di scrittura ilarotragica da Manganelli a Beckett, nell'intersezione di teatro e romanzo, una messa in scena della parola che corrisponde ad uno svuotamento della centralità scenica dell'uomo. Già Carlo Fruttero, introducendo la prima traduzione di En Attendant Godot (prima opera ad essere messa in scena in Italia nel 1954), sosteneva con decisione l'importanza del passaggio dalla prosa al teatro, passaggio che aveva consentito a Beckett di spogliarsi del proprio ingombrante "io", mettendosi finalmente dietro le quinte:

Ora, sulla scena il personaggio che dice "je" per forza di cose non c'è più. Beckett è scomparso dietro le quinte e la parola è tutta alle sue creature. Le quali, dunque, giungono finalmente a persuaderci per il solo fatto che lo possiamo vedere in carne e ossa e constatare che non sono l'autore (Fruttero 1956: 10).

Nei romanzi, Murphy e Molloy non erano di certo Beckett ma la voce dell'autore si sovrapponeva alla loro; mentre il passaggio al palcoscenico consente di dare corpo alle voci dei personaggi, disseminando l'io autoriale in frammenti dialogici che corrispondono ad una molteplicità semantica in continuo movimento. La voce che dice "io" non è identificabile con un singolo personaggio, come accadeva nei romanzi sebbene in maniera del tutto paradossale e non mimetica -, ma viene smembrata in una pluralità di voci che dicono "io", confondendo le identità, i corpi e i ruoli. Bisogna sottolineare, infatti, che la scrittura di En Attendant Godot, avvenuta in pochi mesi dall'ottobre 1948 al gennaio 1949, si colloca proprio durante la stesura della Trilogia romanzesca, subito dopo aver concluso Malone muore e prima di affrontare la totale dissoluzione della parola con L'innominabiles. Si tratta, quindi, di uno slittamento graduale dal romanzo al teatro ma fondamentale per mettere in crisi i generi letterari ed esplorare nuove ibride soluzioni espressive.

Tale ribaltamento di prospettiva è stato messo in evidenza di recente da Gianni Celati, in una intervista rilasciata a Andrea Cortellessa nel 2006, in cui sottolinea come l'intrusione di una gag teatrale all'interno del tessuto narrativo di Molloy sia emblematica dell'operazione di svuotamento dei generi codificati messa in atto da Beckett con dirompente eversione, affermando che «Molloy è una sfottitura buffonesca dei sovratoni del francese letterario», sopratutto negli incisi o negli a-parte e in maniera più estesa «all'inizio, in quel brano su A e B [...], con punte liriche quando descrive il paesaggio, e comiche quando tratta della costipazione nei cani pomeraniani e del classico citoyen che esce a far quattro passi e scoreggiare fuori dalle mura» (Celati 2006: 269). Il passo a cui fa riferimento Celati si configura come una

Cfr. Bertinetti (1994: xvii e segg). V. ovviamente le imponenti biografie beckettiane di Bair (1990) e Knowlson (2001); inoltre il sito italiano dedicato allo scrittore irlandese: www.samuelbeckett.it. 
gag clownesca al rallentatore o da cinema muto (comparabile più a Keaton che a Chaplin), in cui Molloy seduto su un'altura osserva due sconosciuti, identificati con un generico e teatrale A e B, che senza saperlo si stanno per incontrare:

È così che vidi A e B andare lentamente l'uno verso l'altro, senza rendersi conto di ciò che facevano. Era su una strada di una nudità impressionante, voglio dire senza siepi né muri né contorni di sorta, in piena campagna, perché dentro campi immensi delle vacche masticavano, accovacciate e in piedi, nel silenzio della sera (Beckett 1951 [1996]: 6).

In questo paesaggio desolato i due uomini procedono verso lo stesso punto ma ignorano l'uno la presenza dell'altro perché una collinetta impedisce la vista, ma allo stesso tempo sembrano percepire l'occhio esterno che li guarda, anche se non lo danno a vedere. Su quella collinetta infatti è seduto Molloy, l'unico spettatore della scena che si può rendere conto di ciò che sta per accadere, ovvero l'imminente incontro tra i due: «Non avrebbero potuto vedersi, nemmeno alzando la testa e cercandosi con gli occhi, a causa di questo grande spazio, e poi a causa degli avvallamenti del terreno, che rendevano la strada ondulata, non profondamente ma abbastanza, abbastanza» (Beckett 1951 [1996]: 7). A causa della conformazione del paesaggio, A e B non possono prepararsi all'evento e perciò quando si incontrano restano stupiti, bloccandosi l'uno di fronte all'altro: «Ma, forse al rumore dei loro passi, o avvertiti da qualche oscuro istinto, alzarono la testa e si osservarono, per una buona quindicina di passi, prima di arrestarsi, l'uno contro l'altro») (Beckett 1951 [1996]: 7). Lo scambio di sguardi dura pochi interminabili secondi, poi ciascuno va per la sua strada, in una condizione di reciproco disorientamento, ciascuno «preso da un'inquietudine che non era necessariamente la sua, ma di cui in qualche modo lui faceva parte». I personaggi sembrano due attori che vengono spinti sulla scena per caso e controvoglia; ma, rispecchiandosi l'uno nell'altro, essi diventano consapevoli attori della commedia che stanno recitando e di cui Molloy è l'unico spettatore. Si guardano con ansia, compiono gli stessi gesti, come due mimi nel gioco dello specchio, e sono costretti a mettere in discussione la propria soggettività: la loro improvvisa identità, non prevedibile prima di quel casuale momento, è motivo di destabilizzazione per i due uomini, che non avevano mai messo in discussione la propria unicità; mentre nell'osservatore neutrale provoca una reazione di piacevole stupore per la paradossale situazione a cui sta assistendo. L'inquietudine degli attori si trasforma così in una commedia equivoca per lo spettatore, che è la condizione caratteristica di tutto il teatro beckettiano; come ha evidenziato acutamente Guido Guglielmi - nello splendido saggio «L'antiparola della Trilogia», pubblicato su il Verri nel 2002 - affermando che: «Molloy è una maschera di teatro, generato dal teatro e senz'altro fondamento che il teatro. Dunque una figura fantastica. E ogni evento che racconta è un evento fittizio, ogni agire un non agire, e ogni luogo altro luogo» (Guglielmi 2002 [2016]: 122). Il teatro è il fondamento unico che dà voce a personaggi-maschera che non possono avere altra esistenza che quella scenica e che non possono narrare nient'altro che eventi fittizi, privi di referente, attraverso azioni simulate in luoghi indefiniti, che si manifestano il tempo di uno sguardo, quello del pubblico.

Il critico anglo-magiaro Martin Esslin, teorizzatore del "teatro dell'assurdo" con il provocatorio saggio del $1961^{4}$, ha definito questa condizione come "riso dianoeti-

4 Cfr. Esslin (1961 [1975]). Il volume viene recensito anche da Manganelli nel 1963. 
co", che è - secondo la risposta che dà il personaggio di Arséne al protagonista di Watt (1978) - «il riso dei risi, il risus purus, il riso che ride del riso, colui che contempla che saluta lo scherzo più nobile, in una parola il riso che ride - silenzio, prego - di ciò che è infelice» ${ }^{5}$. Ma - puntualizza Esslin - «in Beckett non sono i personaggi ma il pubblico a ridere; l'assenza di dianoia, per quanto terribili possano essere le loro disgrazie, li rende infatti comici e ridicoli. La differenza tra la catarsi dianoetica della tragedia tradizionale e la dianoia del risus purus presente nel teatro di Beckett deriva proprio dalla dislocazione di questa consapevolezza» (Esslin 1986 [1994]: $738)^{6}$. Dunque, nel teatro beckettiano il riso catartico viene generato dallo scarto tra la rappresentazione messa in scena dagli attori, i sintomatici A e B che dialogano inconsapevoli della loro sorte, e lo sguardo dello spettatore che la interpreta, consapevole invece dell'assurdità comica di ciò che sta avvenendo. A e B, anche se il loro destino procede inesorabile sui binari dell'angoscia, non possono essere personaggi tragici, proprio perché non saranno mai consapevoli fino in fondo del loro destino ma resteranno perennemente in un grigio-nero limbo di incomprensione, non solo del mondo ma anche delle parole che utilizzano per interrogarlo. Invece, il pubblico è in grado di comprendere la condizione tragica dei personaggi e dei loro inconcludenti dialoghi, o meglio, dialoghi monologanti, che riempiono il vuoto della scena e della vita. Il pubblico ride delle disavventure che capitano ad A e B - come in una slapstick comedy di Laurel e Hardy con il malinconico volto di Buster Keaton - ma, inoltre, riconosce in loro l'insensata condizione umana. E infatti l'autore irlandese utilizza un'ampia gamma di interpolazioni comiche per interrompere la continuità dell'azione e anche la causalità degli eventi, evitando accuratamente la tentazione della narratività e delle ipotesi ermeneutiche: «Beckett utilizza ogni genere di effetto comico, dal gag-immagine leggibile visivamente come pantomima descritta, al gag verbale sotto forma di commento o ragionamento, al gag-situazione dove due diversi autismi messi a confronto perpetuano un disastroso sfasamento iniziale fino al limite di sostenibilità» (Celati 1997: 91).

Come ha sottolineato Paolo Bertinetti, nell'introduzione al volume che raccoglie tutto il teatro di Beckett, si può riassumere il fulcro di questa paradossale comicità con una battuta di Nell, personaggio di Finale di partita, ovvero la vecchissima madre del protagonista cieco Hamm nascosta dentro un bidone della spazzatura: «Niente è più comico dell'infelicità». Infatti, l'infelice si trova in una condizione tanto disastrosa per sé quanto comica per chi lo osserva dall'esterno, perché cerca di raggiungere qualcosa che non potrà mai ottenere, se non per brevi e impalpabili momenti. L'infelice allora diviene l'emblema della condizione stessa dell'uomo, e di conseguenza «se ridiamo dei personaggi non ridiamo per derisione: ridiamo dell'infelicità umana in quanto ridiamo della beffa più clamorosa di cui è vittima l'umanità, e cioè del fatto che, in ultima istanza, non è possibile cogliere un senso, uno scopo, una finalità nella nostra esistenza» (Bertinetti 1994: xxiii-xxiv). Il cortocircuito si realizza con implacabile precisione, cosicché lo spettatore che ride delle disgrazie altrui, quelle di due sconosciuti e insignificanti attori, A e B, sta ridendo in realtà della propria condizione infelice e priva di scopo, consapevole di riconoscersi in essa.

Beckett (1953 [1967]). La prima traduzione del 1967 è stata poi rivista da Frasca nel 1998 per Einaudi. Riguardo ai temi del mito e della ritualità in Beckett vedi la raccolta di saggi Burkman (a c. di) (1987). 
È quanto accade alla Winnie di Giorni felici che, sepolta in un cumulo di terra, ogni tanto scoppia in un riso forzato senza possibilità di risolversi in una vera e propria gioia, un riso congelato, indice di una vita grigia e insensata:

WINNIE (in un sussurro) Dio. (pausa. Willie ride sommessamente. Dopo un attimo, Winnie si unisce alla risata. Ridono insieme sommessamente. Willie smette di ridere. Winnie continua a ridere per un momento da sola. Willie si unisce a lei. Ridono insieme. Winnie smette di ridere. Willie continua a ridere per un momento da solo. Smette di ridere. Pausa. Voce normale) Ah, ma comunque è stata una gran gioia sentirti di nuovo ridere, Willie, ero sicura che non mi sarebbe successo mai più, che non ti sarebbe successo mai più. (Pausa). Immagino che certa gente potrebbe giudicarci un tantino irriverenti, ma a me non sembra che sia il caso. (Pausa). Non c'è miglior modo per glorificare l'Onnipotente che ridacchiare con lui dei suoi scherzetti, specialmente quelli meno riusciti (Beckett 1961 [1994]: 266).

Winnie è un personaggio svuotato della sua identità e della possibilità di cambiare vita, non è altro che una montagnola di terra e una voce che si lamenta e ride, con sbalzi d'umore improvvisi, come se parlasse le parole di qualcun altro, parole che la attraversano e creano improvvisi brividi emotivi slegati dalla consequenzialità logica del discorso. Winnie ride degli "scherzetti" dell'Onnipotente, dei suoi infantili trick, ma in realtà pronuncia le parole di una voce onnipotente che la domina. Ride di un riso che non sa trattenere e che non riconosce come suo: una risata involontaria, a cui è impedita una liberatoria catarsi.

In questo percorso di annientamento drammaturgico dell'individuo - commenta Aldo Tagliaferri - «la voce divora le personae, l'una dopo l'altra, ma la persona non è che un diaframma tra il vero e il falso, il reale e l'irreale, come falsità cessa di essere quando tramuta nella successiva, ma come verità cessa di essere proprio nel momento in cui incomincia» (Tagliaferri 1979: 99). L'ossessiva catalizzazione sulla voce e la poliedricità dei suoi effetti conduce inesorabilmente verso la scomparsa quasi totale del corpo del personaggio e dell'attore, come accade alla Bocca di Non io, unica protagonista di una soliloquio disintegrato e informe:

BOCCA ... fuori ... dentro a questo mondo... questo mondo... piccola minuscola cosa... prima del tempo... in una dann... - ... cosa? ... bambina? ... sì ... piccola minuscola bambina ... dentro a questa... fuori dentro a questa... prima del suo tempo... a questa dannazione di buco che si chiama... si chiama... non importa... genitori ignoti... mai sentiti... lui sparito... come fumo... (Beckett 1973 [1994]: 431).

Il flusso ininterrotto di parole resta continuamente inconcludente, perché esso dice nient'altro che se stesso: la voce mette in scena tutte le sue variazioni possibili senza parlare di nulla, in uno sfogo che si manifesta come totale ma in realtà si disinnesca da uno sproloquio all'altro. Infatti il personaggio che viene identificato con l'unica parte visibile del corpo, la bocca, «sta tutto nelle parole che pronuncia, nella furia con cui le pronuncia, nella modalità con cui comunica il senso della propria condizione angosciosa, scaricando una valanga di suoni contro il silenzio di un'intera esistenza»; di conseguenza - continua Bertinetti analizzando l'allestimento scenico - «il personaggio e la sua storia sono le sue parole, sono la bocca da cui escono: è per questo che nel buio della sala il riflettore illumina e ci fa vedere solo quella 
porzione di volto» (Bertinetti 1994: xxxvii-xxxviii). La figura umana è ridotta al solo apparato fonatorio, che non serve più a comunicare ma ad emettere prima parole sconnesse e poi suoni, finché diviene mera sineddoche di un corpo («piccola minuscola cosa»), la parodia della comunicazione in quanto "non io", ovvero il simulacro del senso allo stesso tempo «fuori... dentro questo mondo».

$\mathrm{Da}$ questa rapida panoramica su alcuni testi beckettiani si può notare come da $\mathrm{A} \mathrm{e}$ B, i due personaggi muti di Molloy, a Vladimiro ed Estragone che aspettano invano Godot, dalla Winnie di Giorni felici interrata fino al collo, alla Bocca protagonista di Non Io che sproloquia tutta l'insoddisfazione per la propria vita, il riso dianoetico non è quello che accade in scena, o che viene indicato nelle note di regia e attoriali, ma quello del pubblico che assiste alla piéce disorientato e divertito insieme, un riso puro e crudele perché viene strappato inaspettatamente proprio nella condizione angosciosa che accomuna attori e pubblico. E la stessa sensazione che si prova di fronte alla serie dei "papi" eseguita da Francis Bacon tra il 1950 e i primi anni Sessanta - rivisitazione del ritratto di papa Innocenzo X (1605) di Diego Velazquez -, nella quale il volto di un uomo potente viene deformato e ingabbiato dallo stesso ruolo che gli è stato assegnato, spalancando la bocca come in un urlo incontrollabile e muto. Si tratta di una condizione limbica, priva di vie di fuga, che manifesta l'angoscia insita nell'essere umano, anche in colui che possiede un potere smisurato, ma al medesimo tempo, proprio per il fatto che il soggetto è un inaspettato ricettacolo di dolore, come qualsiasi persona, rende l'immagine paradossale, quasi ridicola, decostruendone la codificazione sociale. Il papa soffre come chiunque altro e finalmente, tramite la pittura di Bacon, può dimostrarlo al mondo intero, anche se la catarsi non si compie fino in fondo ma resta paralizzata su quel trono che è simbolo del suo potere. La figura umana esonda i limiti antropomorfi e fa emergere la propria vera natura, che è quella dell'informe materia soggetta alle spinte degli istinti primordiali, senza riuscire ad adattarsi fino in fondo alle convenzioni sociali. Per questa ragione la ricerca artistica di Bacon sull'uomo si sviluppa attraverso una forma dinamica, flessibile, in continua formazione, che corrisponde non ad un destino tragico ma ad una forza che egli definisce "esilarante disperazione", perché - come ha sottolineato Nadia Fusini, mettendolo in relazione a Beckett - è mossa da una destabilizzante vis comica, «un'energia che non teme di frugare in quella terra di nessuno dove l'angoscia e la disperazione si mescolano alla bestemmia, al riso, allo sberleffo, al ridicolo stesso» (Fusini 1994: 66).

In quest'ottica, Giorgio Manganelli è stato uno dei primi, insieme a Carlo Fruttero e Franco Quadri, a recepire la deflagrazione della dicotomia tragedia/commedia attraverso la glorificazione dell'assurdo nel teatro di Beckett. Tutta la produzione manganelliana è permeata di spunti e rivisitazioni beckettiane, come hanno rilevato innanzitutto Aldo Tagliaferri, ripercorrendo la genesi dell'esordio letterario nel 1964 con Hilarotragoedia, e poi Luca Scarlini che ha curato la raccolta di tutto il teatro con il titolo di Tragedie da leggere (2008). Il rapporto di Manganelli con il teatro è molto profondo e, anche se sembra ammirare solo Shakespeare e il teatro elisabettiano da ottimo anglista quale è stato, in realtà è molto attento alle novità a lui contemporanee sia sulla scena che in ambito di critica teatrale. Nei suoi interventi radiofonici e su rivista recensisce numerose pubblicazioni e spettacoli, offrendo anche - come ricorda Scarlini - una particolare e "perplessa" definizione del teatro nell'articolo Cerimonia e artificio pubblicato su il Verri nel 1967, in risposta all'inchiesta promossa da Luciano Anceschi «Cerimonia e artificio» su teatro e letteratura in relazione alla nuova esperienza della neoavanguardia: 
Ho del teatro una idea piuttosto perplessa, e tuttavia eccitante; un sistema di diffidenze, irritazioni e imprecise speranze. Mi irrita che a teatro ci siano attori e pubblico. Cominceremo, dunque, e per tempo a inchiodare porte e finestre [...] Non amo, diffido di, disamo, ho in uggia, in dispetto, detesto il teatro agonistacentrico, inventato per il grande attore, colui che strappa l'applauso a scena aperta (Manganelli 1967 [2000]: 35).

Nonostante l'ostentato disamore, legato soprattutto all'insofferenza per l'agonismo tipico del grande attore, egli è fortemente attratto dalla ritualità teatrale, in quanto «occupazione verbale, gestuale e visiva di uno spazio privilegiato» (Manganelli 1967 [2000]: 36), in cui viene messa in risalto l'interazione tra voce recitante e spazio vuoto delle possibilità sceniche, nonché il gioco di ruolo tra attore, personaggio interpretato e pubblico. Non stupisce infatti che l'esordio pubblico di Manganelli, durante il convegno palermitano del Gruppo 63, sia avvenuto tramite una performance para-teatrale intitolata Hyperipotesi, che rielabora uno dei principi cardine della scrittura di Beckett, ovvero la necessità costante di porre delle ipotesi:

Signori e signore, l'importante è proporre delle ipotesi. Nessuna attività è più nobile di questa, più degna dell'uomo. In primo luogo, in qualsivoglia condizione, senza pausa elaborare ipotesi; in secondo luogo, confortarle di documenti, indizi, argomenti, fenomeni, epifenomeni... Ipotizzare è sano, relaxing... è un'attività euforica ed euforizzante, da week-end, come fondare religioni, concepire generali, merendare con consanguinei... Reperire la appropriata documentazione significa né più né meno, fare l'inventario dell'intero universo. (Manganelli 1975 [2008]: 3)

L'euforica attività dell'ipotizzare è così radicata nel relatore che egli ad un certo punto è costretto ad interrompere la conferenza-spettacolo, perché sente alcuni rumori dietro il sipario che lo distraggono e lo costringono a formulare ipotesi sulla loro natura («Bisogna porsi in situazione di attesa... deliziosa incertezza, sensualità delle ipotesi!»). Dopo aver fatto tutte le supposizioni possibili sull'universo che si trova al di là, la curiosità e lo spavento prendono il sopravvento, così l'oratore saluta il pubblico e scompare dietro al sipario: fine del monologo. Una performance buffonesca ed euforicamente angosciante che produce riso e disorientamento nell'uditorio. Ma per Manganelli la letteratura risiede proprio in quell'infinita disponibilità ad ipotizzare consentita dall'ignoto, come la pagina bianca o il buio iniziale del palcoscenico. In seguito a questo imbarazzante e fallimentare esordio pubblico, egli sviluppa la propria ricerca con Hilarotragoedia (1964), libro nel quale - come ha sottolineato Tagliaferri - l'autore descrive uno "sprofondamento angosciastico" nella natura umana che è anche uno sprofondamento nella testualità di Beckett: infatti si possono rintracciare numerose espressioni verbali e metafore già presenti nella Trilogia e nel teatro che qui vengono ibridate ad una stratificazione linguistica barocca che gioca sugli ossimori e sui paradossi. L'operazione retorica di desacralizzazione e mitizzazione dei generi letterari si fonda sull'emersione di materiali reconditi e di scarto che si annidano nell'oscurità della parola, in linea con tutta una tradizione satirico-umoristica inglese che va da Swift a Beckett; come ha rilevato puntualmente Salvatore Silvano Nigro nel saggio Vita autentica di uno scrittore immaginario: 
«Le parole commerciano con l'ombra. Con la morte, con l'apocalisse già avvenuta, e con l'angoscia che la retorica disciplina, araldizza, e allegorizza, nei modi ilarotragici di un umorismo senza allegria (alla Beckett) e di un umorismo "allucinato e deserto" (non abitato da "uomini e fatti", ma da "simboli e miti", alla Swift)» (Nigro 2006: 130). In questa prospettiva carnevalesca, si ricava l'aggettivo "ilarotragico" che Manganelli riadatta - in chiave beckettiana - sia alle ceneri della commedia che a quelle della tragedia.

A questo proposito, è necessario puntualizzare la distinzione tra la Tragicommedia e la Ilarotragedia, termini apparentemente contigui che in realtà identificano testi di natura assai diversa. Infatti, nel primo caso si intende un componimento teatrale costruito sulla commistione anticlassica tra elementi tragici ed elementi comici, dove quest'ultimi prevalgono conducendo spesso ad un lieto fine; mentre nel secondo caso si intende una forma particolare di commedia (elaborata da Rintone di Taranto nel IV secolo a.C.) caratterizzata da una parodia mitologica, che non prende spunto dal travestimento del mito, ma dalla forma letteraria che il mito aveva assunto nella tragedia. Dunque, la distinzione principale riguarda il principio fondante della coesistenza inversa dei due generi presi in considerazione: l'ibridazione di elementi eterocliti (tragici e comici) si contrappone al travestimento dei generi, in cui il mito tragico veste i panni paradossali del comico.

Il libro d'esordio di Manganelli fa perno proprio su questa distinzione, affrontando il nucleo "angosciastico" dell'uomo attraverso un inconcludente trattatello che simula una coerente analisi di tutte le possibilità che ha a disposizione l'uomo per discendere agli inferi, senza giungere mai ad una vera conclusione (infatti il finale resta sospeso con i due punti che preannunciano un'inesistente soluzione). Il risultato è la parodia di un trattatello secentesco, perché imita lo scheletro del discorso e le formule verbali ma non progredisce secondo una logica puntuale e dialetticamente inappuntabile. Così facendo, la riflessione sulla condizione tragica dell'uomo e sulla tradizione sia mitologica che teologica dell'aldilà si ribalta in una decostruzione del susseguirsi meticoloso delle argomentazioni che dovrebbero costituire il discorso, ed invece lo rendono evanescente e tragicamente ilare.

Questa visione del teatro si evince da una delle strutture dialogiche presenti nella raccolta $A$ e $B$ (1975), nella quale Manganelli porta alle estreme conseguenze, per il contesto italiano, la possibilità di costruire discorsi sul nulla fatti di nulla, ovvero costruiti sul "felice vanverare" - come lo ha definito Grazia Menechella ${ }^{7}$ - del linguaggio sul linguaggio. Nel dialogo che dà il titolo alla raccolta, si affrontano due generici A e B che fanno ipotesi sull'opportunità o meno che avvenga il dialogo tra loro, come due persone che si trovano improvvisamente su un palcoscenico senza sapere per quale motivo ma, nonostante tutto, provano a mettersi in scena come personaggi di se stessi:

A Questo dibattito è ozioso: nessuna forma di saluto ci è congrua.

B Non possiamo congedarci. Sarebbe la fine, alla lettera.

A Né ritrovarci.

B Né augurarci alcunché.

A Pertanto, faremo astrazione da qualsivoglia saluto.

B Mi pare ideologicamente coerente.

Cfr. Menechella (2002); e inoltre Isotti Rosowsky (2007). 
A Tuttavia noi conversiamo; non abbiamo mai fatto altro che conversare.

B Un verbo fatuo, ma proprio.

A Né io posso parlare se non con te.

B Non troveresti altri disposti ad ascoltarci.

A La tua amicizia è l'unico comfort di queste tenebre.

$\mathrm{B}$ Nulla più di un reciproco odio assomiglia a un tiepido caminetto.

A Sebbene sia mio compito infierire su di te e godere del tuo strazio ti debbo confessare la mia leale riconoscenza: se non avessi te da seviziare diventerei scettico sulla mia stessa esistenza.

B E io ti sono grato dell'odio di cui ti sono debitore. Aiuta a passare il tempo.

A Il nostro odio collaborativo sfida un infinito di tedio e giustizia

$[\ldots]$

A Tu credi in Dio?

B Mi aspettavo questa domanda. È il riflusso della crisi. Rassicurati: il teologo sono io e ti dico: finché non crei, io credo in te.

A Ma se non credo, se resto frustrato nella mia vana onnipotenza, io cesso di credere in me. (pausa.) Divento ateo.

$\mathrm{B}$ Volevo sentirtelo dire; te ne sono grato. Ora la nostra società è perfetta. Potremo anche cessare definitivamente la nostra conversazione. Vorrei suggerirti una formula.

A Dimmi.

B Io credo in Dio; ma dio è ateo. (pausa.) Ti va?

A (con implicito, pacifico sorriso) Mi va. (Manganelli 1975: 15-23)

I due non meglio identificati A e B - come accade in Molloy - sono personaggi vuoti che riempiono il vuoto delle loro esistenze attraverso un dialogo irrisorio e allo stesso tempo universale, sorridendo della loro comune condizione e della soluzione finale («Io credo in Dio; ma dio è ateo») senza possibilità di catarsi. Come ha ben illustrato ancora Tagliaferri, la vacuità connaturata allo spazio teatrale diventa allo stesso tempo il luogo ideale per lasciare libero sfogo alla formulazione di ipotesi su altre ipotesi ma anche non-luogo angoscioso che corrisponde alla condizione di disorientamento caratteristica dell'uomo moderno:

Le variazioni sul tema del nulla nella sticomitia di A e B mantengono una decisa connotazione beckettiana, come del resto la collocazione delle anonime voci che le enunciano su un palcoscenico "completamente buio". Manganelli [...] riprenderà la metafora esemplare, già presente nella Trilogia, della persona incarcerata per una colpa ignota, o forse non commessa, e identificherà il non-luogo in cui, malgrado tutto, sopravvive il suo personaggio, con una palude, come quella già apparsa in Molloy. [...] Manganelli da parte sua rielaborò questa estrema metafora dopo aver assimilato la concezione del fallimento della parola propugnato da Beckett, quando, lasciatosi alle spalle l'insalubre mondo accademico, si sentì libero di "fallire" a modo suo (Tagliaferri 1998: 57).

Manganelli condivide con Beckett proprio la necessità del fallimento, un fallimento premeditato e allestito in ogni minimo particolare: una messa in scena della sconfitta della logica del discorso che è anche una liberazione delle sotterranee potenzialità semantiche del linguaggio. Tale paradossale missione viene perseguita al contrario smascherando la vacuità stessa dell'obiettivo a cui si ambisce. In questa 
prospettiva, le caratteristiche peculiari del teatro (la voce, la scena, l'uditorio) mettono ancor più in evidenza l'insensatezza delle categorie e delle codificazioni entro cui è ristretta la parola letteraria, perché la dialogicità insita nei testi per il teatro implica un elemento altro a cui rivolgersi - un personaggio o il pubblico silenzioso nella sala o una entità ignota che si nasconde dietro il sipario - che duplica, rispecchia e deforma il significato delle parole pronunciate sulla scena, aumentando a dismisura l'espansione semantica oltre il limite del nonsense. Ogni testo teatrale di Manganelli, ogni "tragedia da leggere", si configura infatti - lo ha sottolineato Scarlini - come «colloquio cerimoniale con l'altro da sé, sia esso il potere o l'angoscia [...], in una continua tensione dialettica tra elementi diversi, su un palcoscenico più sognato e immaginato che effettivamente agito» (Scarlini in Manganelli 2008: 1xi).

Massimo esempio della messa in scena cerimoniale dello spazio teatrale si ha con il dialogo a più voci che si svolge In un luogo imprecisato, testo teatrale scritto per Radio Rai nel 1974, nel quale quattro voci anonime sono collocate in un luogo altrettanto anonimo e cominciano a domandarsi chi sono e per quale motivo si trovano lì. Esse discutono delle possibili identità che potrebbero caratterizzarle, assumendone sempre di nuove in base alle loro inconcludenti supposizioni (re, regine, generali, imperatori...), e si interrogano sulle caratteristiche di quello spazio buio che le contiene ma che non fornisce punti di riferimento (Manganelli infatti gioca anche con l'evanescenza dell'etere radiofonico). Odono solo il suono delle loro parole e di qualche rumore non identificabile, rumori che riverberati dai loro dialoghi in una situazione di stallo producono un senso di totale disorientamento ma consentono anche la proliferazione di una moltitudine di ipotesi. Si viene a comporre così un dialogo intrecciato di voci che fanno ipotesi sulla propria natura, ipotesi che si innestano l'una sull'altra ma non possono essere verificate nella totale oscurità. L'incipit è rivelatore della prevalenza del senso dell'udito, come unico e solo mezzo per un tentativo di definizione del luogo:

Si sente un rumore regolare come di chi respiri tra sonno e veglie [...]

Si ode anche come l'inizio di un parlottìo indefinito, inafferrabile, di chi parli nel sogno o con qualcuno [...]

Si ode un rumore brusco; qualcuno bussa alla porta. (Manganelli 1975 [2008]: 105)

Il ritmo dialogico del testo è dato dal continuo rinnovarsi di supposizioni sospese, che ad ogni nuovo rumore, sussurro o voce cambiano direzione, vengono riformulate, si intersecano con le precedenti oppure le sostituiscono cancellandole. Il buio totale in cui si trovano le voci cancella i punti di riferimento, lasciandole in balia dell'incertezza, di quel frenetico ipotizzare possibili mappature e impraticabili vie d'uscita o d'entrata, che non conduce ad alcuna soluzione.

In ogni voce si coglie la preoccupazione per l'assenza di identità ma anche la totale disponibilità ad essere attraversata da identità plurime e provvisorie che si vanno costruendo nel dialogo con le altre voci; come accade alla quarta voce, che dopo aver bussato a lungo alla porta giunge per ultima in quel luogo imprecisato che non è né un dentro né un fuori:

Chi sono? È strano: sono perfettamente lucido, ma ho un'idea insieme intensa e imprecisa di chi sono; credo che dipenda dal fatto che non so dove sono. Sì, ho faticato a entrare, e ho anche avuto paura. Deve essere stato durante la paura, dopo quei colpi, che mi sono un po' smarrito... (Manganelli 1975 [2008]: 112) 
I personaggi sono ridotti ai minimi termini, non-io dialoganti, meri apparati fonatori, ma in questo modo aumentano a dismisura la loro disponibilità alla metamorfosi, alla frammentazione degli "io", all'accelerata oscillazione semantica a cui sottopongono le parole che pronunciano senza un orizzonte di riferimento definito ma costantemente intercambiabile. Perciò, è il linguaggio ha costruire se stesso e il luogo che lo ospita, ma non è di nessun aiuto alle voci che tentano di dare un significato alla loro condizione. La parola prende vita dal sussurro, declinando infinite ipotesi, ma allo stesso tempo muore nella vastità dei campi del possibile, divenendo vuoto blaterare nel vuoto, rovello su ipotetici sviluppi che mette in scena se stesso in quanto movimento del linguaggio: e dunque «sur un plan théorique, la piéce pourait donc s'envisager comme une mise en scéne du langage par lui-meme» (Denize 2008:189) ${ }^{8}$. Questa affermazione vera sul piano teorico viene rafforzata dall'aspetto performativo, che mette in evidenza con maggiore precisione il valore autocompositivo del linguaggio, ovvero il linguaggio che mette in scena se stesso.

Infatti, In un luogo imprecisato è stato ideato come dramma radiofonico per il terzo programma della Rai, e dunque esso sfrutta appieno le possibilità del mezzo, divenendo una sorta di mise en abyme del mezzo stesso. Il luogo approssimativo, in cui si affollano le voci incerte sulla loro identità e sulla loro collocazione spaziale, si può identificare con l'oggetto radio, dal quale provengono voci che appartengono a corpi intangibili e non collocabili in un luogo determinato, ma solo ipotizzabile. È su questa ambiguità che Manganelli gioca con la voce di Carmelo Bene, offrendogli di interpretare tutte le voci maschili (l'unica voce femminile è affidata a Lydia Mancinelli), perché l'abilità vocale dell'attore salentino permette una pluralità di toni che ingannano e disorientano l'ascoltatore, il quale viene convinto a credere nella presenza di un gruppo di persone in quel luogo "imprecisato", pur non potendole associare ad alcun corpo. Le voci radiofoniche sono voci assolute, prive di corporalità, collocate in una dimensione che non è dato conoscere, ma proprio per questo chi ascolta può cogliere con particolare intensità le variazioni di tono e l'energia che emanano.

L'indeterminatezza identitaria dei personaggi e quella spaziale dei luoghi è la stessa che ritroviamo nell'opera beckettiana. È stato il filosofo francese Alain Badiou a mettere in evidenza la funzione fondamentale dell'alterità in Beckett, indagando la peculiare tensione verso l'altro dei personaggi solitari presenti in tutta la sua opera, quel increvable désir di confrontarsi con l'altro, di "essere-due", di moltiplicare la propria malferma identità attraverso il teatro. Così l'attenzione di Beckett è rivolta a «proiettare, sullo sfondo dell'essere anonimo, l'affaccendarsi dell'umanità plurale, catalogandone gli atteggiamenti e redigendo il repertorio delle sue funzioni», in testi che si caratterizzino come laboratori purgatoriali per osservare le differenti reazioni dell'uomo di fronte a se stesso, di fronte ad un destino ignoto:

Quei testi sono delle commedie umane in cui la diversità delle figure sociali e soggettive è sostituita dall'inventario, che si vuole esaustivo, di tutto ciò che l'esistenza contempla come possibilità essenziali. Ma nel contempo sono anche delle divine commedie, poiché la volontà di procedere alla catalogazione completa di azioni e situazioni [...] presuppone un luogo immutabile, separato dalla realtà empirica, una sorta di no man's land sospesa tra la vita e la morte (Badiou 2008: 39-40).

Sui temi della vocalità in scena cfr. anche Camplone (2014). 
Ogni generico personaggio A necessita di un altrettanto generico B per farsi attraversare da un pluralità di identità, per prestare la propria voce alla molteplicità di voci che corrispondono all'inventario delle figure umane che popolano quel limbo che è la commedia umana della quotidianità. Ogni loro interazione consente di riempire l'ineludibile vacuità della scena teatrale, ma allo stesso tempo consente la rifrazione del discorso tra palcoscenico, platea e dietro le quinte in una incontrollabile proliferazione semantica. Proprio per questa capacità da parte di Beckett di sfruttare le illimitate risorse delle coppie teatrali - anche le dualità codificate dalla tradizione scenica -, portando alle estreme conseguenze la funzione dialogica fino ad una moltiplicazione esponenziale delle voci, Badiou giunge ad affermare che lo scrittore irlandese «è stato indubbiamente il solo grande scrittore del Novecento a collocarsi nella tradizione del teatro comico d'autore», non solo per la rivisitazione della contrapposizione tradizionale tra due attori senza scadere in trivialità e facili gags, «ma soprattutto la ferma ostinazione dei personaggi nel perseverare nella loro condizione, a schierarsi, nonostante venti e maree contrarie, dalla parte del principio di piacere, della forza vitale, che le circostanze procurano ad ogni istante di ridurre all'impossibilità e all'illegittimità» (Badiou 2008: 59-60).

In questo senso, credo di poter affermare che il riso dianoetico del teatro beckettiano, in quanto mancata catarsi dei personaggi sulla scena, corrisponda alla definizione di riso "ilarotragico" proposta da Manganelli nell'omonimo trattatello sulle diverse modalità dell'uomo di discendere dentro il proprio inferno, poiché lo svuotamento delle strutture codificate della tragedia e della commedia determina uno spazio scenico totalmente vuoto, nel quale qualsiasi discorso può accadere senza condurre ad alcuna soluzione dialettica. I personaggi si mostrano sulla scena per quello che sono, ovvero figure vuote prive di identità, nient'altro che corpi deformi o mere voci che danno vita a discorsi sconnessi senza limite, giungendo fino all'estremo ribaltamento dell'angosciosa condizione umana in buffonesca commedia alla ricerca dell'irraggiungibile senso della vita. Ne è un chiaro esempio il testo teatrale Il personaggio - pubblicato postumo da Luca Scarlini nel 2002 -, che si configura come un monologo "abitato" da due e addirittura più voci, che fanno riferimento però tutte alla stessa figura scenica, assumendo sempre nuove identità: all'inizio un musicologo esperto del Don Giovanni di Mozart, la cui trama si intreccia e sdoppia ne I Menecmi di Plauto, scivolando verso la fine attraverso i personaggi de L'avaro di Moliére, in un continuo gioco iperletterario di sdoppiamenti e sradicamenti. Dopo il primo lungo monologo mozartiano, il personaggio si sdoppia in due personaggi identici, 1 e 2, ovvero due Sosia, due "infimi" Sganarello:

1 - Dici a me?

2 - Oh, no, signore; non oserei parlarvi a quel modo.

1 - Non dici a me?

2 - No, davvero, io dico a me, solo a me.

1 - Parli solo con te?

2 - E con chi dovrebbe mai parlare una bassezza pari mio?

1 - Tu sei infimo?

2 - Molto infimo, signore. La mia infimità è tale, che per parlare con me debbo parlare a voce molto alta. Eppure io in genere mi sono vicino.

1 - Dunque, tu non parli che con te stesso; ma ti sopporti?

2 - A dire il vero, io nemmeno parlo con me stesso; quando mi parlo, lo faccio per zittirmi (Manganelli 2008: 255). 
Dal personaggio 1 nasce la voce di un personaggio 2, con il quale prende avvio un dialogo insensato, che ha luogo senza avere necessità. Le due voci sono così irrisorie che non possono far altro che discorrere della loro "infimità", perché non c'è nessuna vera esigenza di dialogare, se non quella, altrettanto "infima", di condividere la propria alterità, di rispecchiarsi l'uno nell'altro per constatare l'effimera consistenza di un qualsiasi personaggio sulla scena. Una "infimità" dialogante che risulta ancora definitivamente paradossale nell'atto unico manganelliano Monodialogo, pubblicato sulla rivista Grammatica del gennaio 1967, in cui le voci emergono e allo stesso tempo si dissolvono solo in funzione della perpetuazione della menzogna: «A - Ma tu mediti. B - Certamente: inoltre tramo, complotto, m'ingegno. Oppongo il nulla al niente, congiuro e mento per togliere il potere dalle mani del nessuno e consegnarlo al nessuno, insidio l'inesistente, e dico menzogne che nessuno può distinguere dalla verità...» (Manganelli 1975 [2008]: 62).

Si può individuare una stretta corrispondenza con le tele di Francis Bacon, nelle quali - lo ha sottolineato Michel Leiris, pur indugiando fin troppo sul valore tragico della sua pittura - le figure umane messe in scena in spazi uniformi e destabilizzanti, che rievocano in qualche modo il palcoscenico o la pista di un circo, «sono consegnate allo spettatore [...] esattamente per quel che sono, in un assoluto presente non appannato dalla speranza né dalla nostalgia, e senza che si debba cercare un simbolismo»; per questa ragione, l'arte del pittore irlandese è caratterizzata da una amoralità totale e «non è gravata da alcun messaggio e non pretende né di commentare le tristezze, né di riflettere l'intima natura delle cose: l'immagine uscita dalla sua testa e dalle sue mani deve affermare la sua presenza, nient'altro» (Leiris 1981 [2001]: $48)^{9}$. Siamo di fronte a quella operazione di disintegrazione e svuotamento della identità dei personaggi sulla scena che di recente Gabriele Frasca ha definito - riprendendo il titolo di un racconto di Beckett - come uno "spopolatoio" 10 , ovvero un non-luogo certamente privo di una connotazione specifica e di una codificazione linguistica ma al medesimo tempo gremito di corpi e di voci che si affollano, si rincorrono, attraversano lo stesso personaggio e lo "spopolano", lo "spolpano" fino ad un ilare e catartico sfinimento.

Si tratta, tanto per i due autori irlandesi quanto per Manganelli, di quella estetica del "disfacimento" - come l'ha chiamata Gontarski in relazione all'ultima fase dell'opera beckettiana - che va alla ricerca di strutture aperte che accolgano il caos senza conferire una forma prestabilita e definitiva, anzi seguendo l'infinità indecidibilità che lo caratterizza. È per questa ragione che, infine, è possibile ricondurre i tre autori, pur nelle loro specificità, entro la frastagliata categoria di "ilarotagici", in cui la tragica vacuità dei destini umani è affrontata non come annientamento definitivo ma come apertura verso il rimosso, l'inesplorato, l'indistinto. La letteratura si configura allora come spazio "imprecisato" in cui gli opposti coincidono, alto e basso si ribaltano ma senza una paternalistica e inoffensiva ironia, perché - come precisa ancora Guglielmi: «Non il sublime visto dal basso, dal solido suolo della prosa, e ironizzato, che fraintende e idealizza la realtà, scambiando il reale con l'immaginario, l'essere con il dover essere, ma il basso della (bruniana) coincidentia oppositorum, che disordina, degerarchizza, disumanizza le cose. Un basso disambientato, straniato, sottratto alla misura, al di fuori delle convenzioni di basso e alto. (Un su-

Sull'affinità tra Beckett e Bacon v. almeno Hale (1993: 95-102); e in ambito italiano Fusini (1994).

10 Cfr. Frasca (2014); v. inoltre Frasca (1988). 
blime del comico) » (Guglielmi 2002 [2016]: 123-124). La parola "ilarotragica" non solo sprofonda negli abissi del comico portando giù con sé anche il tragico ma fa emergere il sublime del comico in tutta la sua "esilarante disperazione".

\section{Riferimenti bibliografici}

Alfano, Giancarlo / Cortellessa, Andrea (a c. di) (2006): Tegole dal cielo. L' "Effetto Beckett" nella cultura italiana, vol. I, Roma, Antalia.

Alfano Giancarlo (2010): Paesaggi, mappe, tracciati. Cinque studi su letteratura e geografia, Napoli, Liguori.

Badiou, Alain (2008): Beckett. L'inestinguibile desiderio, Genova, il Melangolo.

Bair, Deirdre (1990): Samuel Beckett: una biografia, Milano, Garzanti.

Baldacci, Alessandro (2010): Le vertigini dell'io. Ipotesi su Beckett Bachmann Manganelli, S. Maria Capua Vetere, Ipermedium.

Beckett, Samuel (1953 [1967]): Watt, Paris, Olympia Press. [Trad. it. di C. Cristofolini, Milano, Sugar\&Co].

Beckett, Samuel (1951 [1996]): Molloy, Paris, Les Editions de Minuit. [Trad. it. di A. Tagliaferri, Molloy in S. Beckett, Trilogia, Torino, Einaudi, pp. 5-192].

Beckett, Samuel (1994): Teatro completo: drammi, sceneggiature, radiodrammi, pièces televisive, a c. di P. Bertinetti, traduzioni di C. Fruttero et alii, Torino, Einaudi-Gallimard.

Bertinetti, Paolo (1994): «Beckett, o la compressione della forma», introduzione a S. Beckett, Teatro completo: drammi, sceneggiature, radiodrammi, pièces televisive, a c. di P. Bertinetti, traduzioni di C. Fruttero et alii, Torino, Einaudi-Gallimard.

Burkman, Katherine H. (ed.) (1987): Myth and ritual in the plays of Samuel Beckett, London and Toronto, Fairleigh Dickinson University press.

Camplone, Ilaria (2014): «Il teatro di Giorgio Manganelli, "un luogo imprecisato"», in G. Baldassarri / V. Di Iasio / P. Pecci / E. Pietrobon / F. Tomasi (a c. di), La letteratura degli italiani 4. I letterati e la scena. Atti del XVI Congresso Nazionale Adi, Sassari-Alghero, 19-22 settembre 2012, Roma, Adi editore, pp. 1-11.

Celati, Gianni (1997): «L'interpolazione e il gag», in S. Colomba (a c. di), Le ceneri della commedia. Il teatro di Samuel Beckett, Roma, Bulzoni, pp. 83-105.

Celati, Gianni (2006): «Pensieri su Beckett», in G. Alfano / A. Cortellessa (a c. di), Tegole dal cielo. L' "Effetto Beckett” nella cultura italiana, vol. I, Roma, Antalia, pp. 269-271.

Denize, Joseph (2008): «Oralité et ènonciation dans "In un luogo imprecisato" de Giorgio Manganelli», in C. Faverzani (éd.), "Je suis l'écho...". L'ecriture et la voix. Hommage offert à Giuditta Isotti Rosowsky, Saint-Denis, PUV, pp. 181-197.

Esslin, Martin (1961 [1975]): The Theatre of the Absurd, Garden City, Doubleday. [Trad. it. di R. De Baggis e M. Trasatti, Il Teatro dell'assurdo, Roma, Abete].

Esslin, Martin (1986 [1994]): «Dionysos’ Dianoetic Laugh», in J. Calder (ed.), As No Other Dare Fail, London, Calder, pp. 15-23. [Trad. it. di E. Cassarotto, «Il riso dianoetico di Dioniso», in S. Beckett, Teatro completo: drammi, sceneggiature, radiodrammi, pièces televisive, a c. di P. Bertinetti, traduzioni di C. Fruttero et alii, Torino, Einaudi-Gallimard, pp. 736-743].

Frasca, Gabriele (1988): Cascando. Tre studi su Samuel Beckett, Napoli, Liguori.

Frasca, Gabriele (2014): Lo spopolatoio. Beckett con Dante e Cantor, Napoli, d'If.

Fruttero, Carlo (1956): «Introduzione», in S. Beckett, Aspettando Godot, Torino, Einaudi, pp. 5-13. 
Fusini, Nadia (1994): B \& B: Beckett e Bacon, Milano, Garzanti.

Gontarski, Stanley (1997): «L'estetica del disfacimento», in S. Colomba (a c. di), Le ceneri della commedia. Il teatro di Samuel Beckett, Roma, Bulzoni, pp. 107-133.

Guglielmi, Guido (2002 [2016]): «L'antiparola della Trilogia», il Verri, 18, pp. 13-39. [Ora in G. Gugliemi, Critica del nonostante, a c. di V. Cuccaroni, prefazione di N. Lorenzini, Bologna, Pendragon, pp. 113-144].

Hale, Jane Alison (1993): «Framing the Unframable: Samuel Beckett and Francis Bacon», in M. Buning / L. Oppenheim (ed.), Beckett in the 1990s, Amsterdam, Rodopi, pp. 95-102.

Isotti Rosowsky, Giuditta (2007): Giorgio Manganelli: una scrittura dell'eccesso, Roma, Bulzoni.

Knowlson, James (2001): Samuel Beckett: una vita, a c. di G. Frasca, Torino, Einaudi.

Leiris, Michel (1981 [2001]): «Bacon le hors-la-loi», Critique, 408, maggio, pp. 519-525. [Trad. it. di F. Nicolao e R. Rossi, «Bacon il fuorilegge», in M. Leiris, Francis Bacon, Milano, Abscondita, pp. 45-60].

Manganelli, Giorgio (1962 [2004]), «Murphy di Beckett», L’illustrazione italiana, 12, dicembre, pp. 72-73. [Ora in G. Manganelli, Letteratura come menzogna, Milano, Adelphi, pp. 99-102].

Manganelli, Giorgio (1967 [2000]), «Cerimonia e artificio», il Verri, 25, marzo, pp. 80-81. [Ora in G. Manganelli, Cerimonie e artifici, a c. di L. Scarlini, Salerno, Oedipus, pp. 36-37].

Manganelli, Giorgio (1975 [2008]): A e B, Milano, Rizzoli. [Ora in G. Manganelli, Tragedie da leggere, a c. di L. Sacarlini, Milano, Bompiani].

Manganelli, Giorgio (2008): Tragedie da leggere, a c. di L. Scarlini, Milano, Bompiani.

Menechella, Grazia (2002): Il felice vanverare: ironia e parodia nell'opera narrativa di Giorgio Manganelli, Ravenna, Longo.

Nigro, Silvano Salvatore (2006): «Vita autentica di uno scrittore immaginario», in M. Belpoliti /A. Cortellessa (a c. di), Giorgio Manganelli, Riga, 25, pp. 130-132.

Scarlini, Luca (1996): Un altro giorno felice: la fortuna dell'opera teatrale di Samuel Beckett in Italia, 1953-1996, Firenze, Maschietto e Musolino.

Scarlini, Luca (2008): «Dialogo notturno: un palcoscenico per Giorgio Manganelli», introduzione a G. Manganelli, Tragedie da leggere, a c. di L. Scarlini, Milano, Bompiani, pp. ix-lxi.

Tagliaferri, Aldo (1979), Beckett e l'iperdeterminazione letteraria, Milano, Feltrinelli.

Tagliaferri, Aldo (1998), «Intorno alla genesi di Hilarotragoedia di Giorgio Manganelli», il Verri, XLII (6), pp. 25-32. 